\title{
Sensitivity to CPT-11 and platinum derivatives of stage II/III node-positive gastric cancer with occult neoplastic cells in lymph node sinuses
}

\author{
Masaya Mukai ${ }^{1)}$, Shinkichi Sato ${ }^{2)}$, Hiromi Ninomiya ${ }^{2)}$, Kanako Wakui ${ }^{2)}$, Nobukazu Komatsu ${ }^{2)}$, \\ Naruaki Matsui $^{2)}$, Masato Nakamura ${ }^{3)}$, Kyoji Ogoshi ${ }^{4)}$, and Hiroyasu Makuuchi ${ }^{4)}$ \\ ${ }^{1)}$ Tokai University Hachioji Hospital, Department of Surgery, ${ }^{2)}$ Tokai University Oiso Hospital, Department of Pathology, \\ ${ }^{3)}$ Tokai University Hachioji Hospital, Department of Pathology, ${ }^{4)}$ Tokai University School of Medicine, Department of Surgery
}

\begin{abstract}
Abbreviations ONCs; occult neoplastic cells, LN; lymph node, 5-FU; 5-fluorouracil, LV; leucovorin, CPT-11; irinotecan, Topoisomelase-1 inhibitor; topo-1, L-OHP; oxaliplatin, CDDP; cisplatin, Bax; bcl-2 associated X, ERCC-1; excision repair cross-complementation group 1, TS; thymidilate synthase, DPD; dihydropyrimidine dehydrogenase, ITCs; isolated tumor cells, RG; recurrence group, N-RG; non-recurrence group
\end{abstract}

\begin{abstract}
In this study, immunohistochemistry was used to detect Topo-1 and Bax/ERCC-1 expression in patients who had lymph node metastasis of stage II/III gastric cancer with occult neoplastic cells (ONCs) in their lymph node sinuses. In addition, tumor sensitivity was compared between CPT-11 and platinum agents. In the recurrence group (RG) $(n=28)$, immunohistochemistry revealed high Topo-1 expression in 3 patients (10.7\%) and low expression in 25 patients (89.3\%), while there was high expression in 1 patient $(11.1 \%)$ and low expression in 8 patients $(88.9 \%)$ from the non-recurrence group $(\mathrm{N}-\mathrm{RG})(\mathrm{n}=9)$ (Not significant; N.S.). Immunostaining showed high Bax/low ERCC-1 expression in 11 patients (39.3\%) from the RG and other patterns of expression in 17 patients (60.7\%), while high Bax/low ERCC-1 expression was observed in 3 patients $(33.3 \%)$ from the N-RG and other patterns were noted in 6 patients $(66.7 \%)$ (N.S.). There was a significant difference between the rate of high-Bax/low-ERCC-1 expression and high Topo- 1 expression in the RG $(\mathrm{p}=0.0136)$. These results suggest that platinum derivatives such as CDDP are more effective than CPT-11 in both RG and N-RG of stage II/III gastric cancer with ONCs.
\end{abstract}

Keywords Gastric cancer, occult neoplastic cells, CPT-11, Topoisomelase-1 inhibitor, platinum agents, Bax, ERCC-1, isolated tumor cells

(Received June 19, 2007; Accepted July 29, 2007)

\section{Introduction}

Tumor recurrence/metastasis to the lungs and liver after radical resection of gastrointestinal cancer is presumed to be related to the survival and proliferation in distant organs of residual cancer cells that circulate during the perioperative period and escape from the host's immune defenses ${ }^{1-4)}$. There have been many reports suggesting a close relationship between the recurrence/ metastasis of cancer and the detection of cytokeratinpositive occult neoplastic cells (ONCs) in the sinuses of lymph nodes distant from the primary tumor ${ }^{5-8)}$. ONCs can be counted by staining these malignant cells that become trapped in the microcirculation of the lymph nodes (LNs), using a simple immunostaining method (patent

Correspondence to: Masaya Mukai, MD, Tokai University Hachioji Hospital, Department of Surgery, Ishikawa-cho 1838, Hachioji, Tokyo, 192-0032, JAPAN FAX:+81-426-39-1112 TEL: +81-426-39-1111 (Ex. 5007) E-mail: mukai.masaya@ hachioji-hosp.tokai.ac.jp pending since 2002 in Japan) $)^{2,3)}$. Free cancer cells are mentioned as isolated tumor cells (ITCs) in the breast cancer section of the sixth version of the TNM classification published in 2002 $2^{9)}$. ITCs are defined as cells/clusters with a diameter of $\leq 0.2 \mathrm{~mm}$ that have not penetrated the walls of vessels or lymphatic sinuses. Larger clusters of floating cancer cells are also observed sometimes, but the significance of such clusters remains unclear. Solitary ONCs in the sinuses of LNs distant from the primary tumor can only be detected by cytokeratin immunostaining. ONCs are also defined as including small clusters of $\leq 10$ cells, while malignant micro-aggregates contain $>10$ cells $^{2,3,10}$. ONC clusters can theoretically cause recurrence/metastasis in any organ, and should be differentiated from micrometastases $(0.2 \mathrm{~mm}$ to $2 \mathrm{~mm}$ in diameter) anchored in LNs or from ITCs, since ONCs seem to be occult systemic metastases with a higher malignant potential ${ }^{10-12)}$.

When postoperative chemotherapy is performed for advanced gastric cancer and colorectal cancer, the 
combination of 5-fluorouracil + leucovorin (5-FU/LV) and/or cisplatin (CDDP) and/or irinotecan hydrochloride (CPT-11: a topoisomerase-1 inhibitor with a novel mechanism of action) has recently come into widespread use $^{13-17)}$. Platinum derivatives such as CDDP generally inhibit DNA synthesis by forming DNA-platinum adducts. Like 5-FU, their efficacy is greatly influenced by the sensitivity of the target tumor. Indeed, the efficacy of platinum compounds has been reported to be related to the following factors: 1) an increase of DNA excision repair due to up-regulation of nuclear excision repair genes such as excision repair cross-complementation group-1 (ERCC-1) gene; 2) increased expression of anti-apoptotic genes such as those of the Bcl-2 family coupled with the P53 tumor suppressor gene; and 3) increased excretion from tumor cells by the CDDP/glutathione complex and ATP-binding cassette transporter ${ }^{18-22)}$. Up-regulation of ERCC-1 gene expression is reported to be related to the acquisition of tumor resistance to platinum derivatives such as CDDP and L-OHP ${ }^{18,20,21)}$. It has also been reported that resistance to apoptosis due to decreased expression of the Bcl-2-associated X (Bax) gene has a marked influence on the acquisition of resistance to platinum compounds $^{19)}$. These reports suggest that tumors with high Bax/low ERCC-1 expression may be more sensitive to platinum derivatives ${ }^{23-25)}$.

However, there have been no previous immunohistochemical investigations of topoisomerase-1 (Topo-1) and Bax/ERCC-1 expression in the metastatic LNs of patients with node-positive stage II/III gastric cancer who are also positive for ONCs. In the present study, the sensitivity of LN metastases to anticancer agents such as CPT-11 and platinum derivatives was investigated by immunohistochemical determination of Topo-1 and Bax/ERCC-1 expression in patients who had node-positive stage II/III gastric cancer with ONCs, and tumor sensitivity was compared between CPT-11 and platinum agents such as CDDP.

\section{Materials and Methods}

A total of 75 patients with stage II/III LN-positive primary gastric cancer underwent curative resection during the 13 years from 1987 to 2001 and met the following criteria: 1) complete medical records were available and the presence/absence or recurrence and survival could be confirmed; and 2) at least 10 LNs were harvested that showed no metastasis on routine H.E. staining. Among the 75 patients, 37 were registered in the database of the Occult Neoplastic Cells Research and Study Group. In these 37 patients, the presence of ONCs was confirmed by cytokeratin immunostaining (AE1/AE3; Fuji Chemical Industries, Ltd., Japan) ${ }^{26-29)}$.

The 37 patients were divided into a recurrence group (RG) $(n=28)$ who showed recurrence/metastasis within five years of surgery and a non-recurrence group $(\mathrm{N}-\mathrm{RG})(\mathrm{n}=9)$ without recurrence after at least five years. Immunostaining of LNs for Topo-1 and Bax/ERCC-1 was done by the routine indirect immunoperoxidase method using a monoclonal anti-Topo-1 antibody as the primary antibody (Topo-1; 1D6, Dako, Denmark), as well as polyclonal anti-Bax and anti-ERCC-1 antibodies (Bax; PC66T, Calbiochem, Germany, ERCC-1; SPM243, Spring Bioscience, USA). Minor modifications were applied to the staining technique, such as retrieval of antigenicity ${ }^{30-33)}$. Positive tumor cells were counted at a high power and staining was classified into the following four grades: $0 \%(-),<5 \%(+), \geq 5 \%$ to $<30 \%$ $(++)$, and $\geq 30 \%(+++)$. Both $(-)$ and $(+)$ were defined as "low expression," while $(++)$ and $(+++)$ were defined as "high expression" (Figures 1, 2) $)^{30-33)}$. To determine

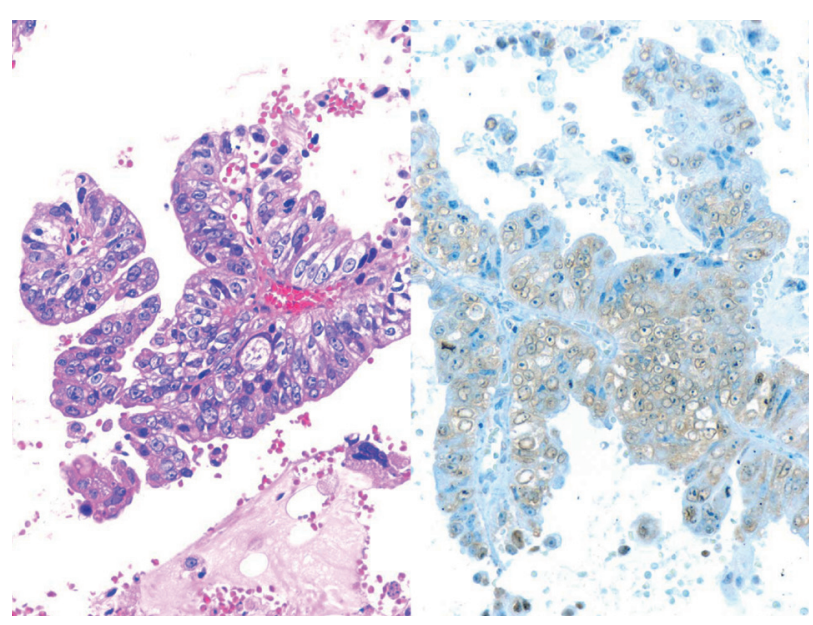

Fig. 1 Immunohistochemical staining for topoisomerase-1 (Topo-1) (high expression; +++) in the metastatic lymph node of a patient with recurrence of stage III gastric cancer and positive occult neoplastic cells (left: H.E. stain, $\times 200$, right: Topo-1, ×200).

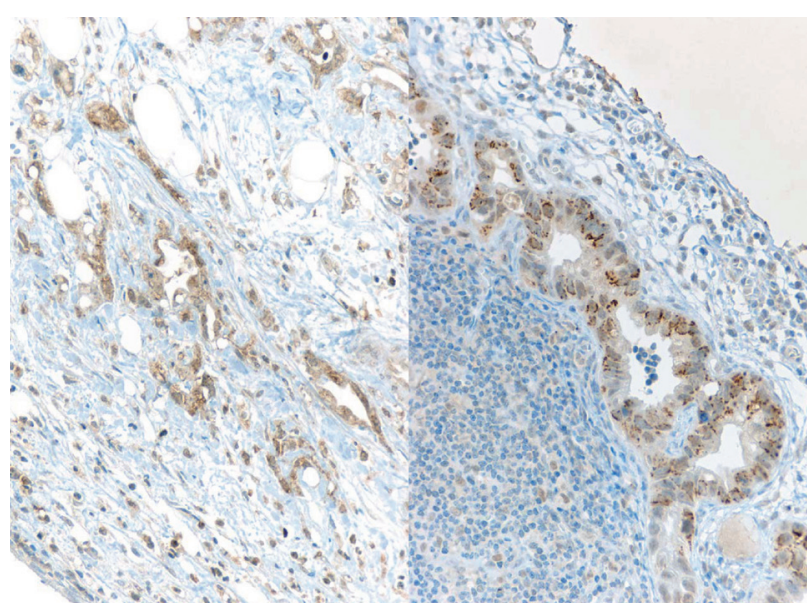

Fig. 2 Immunohistochemical staining for Bcl-2-associated $\mathrm{X}(\mathrm{Bax})$ and excision repair cross-complementation group 1 (ERCC-1) (high expression; +++) in the metastatic lymph node of a patient with recurrence of stage III gastric cancer and positive occult neoplastic cells (left: Bax, ×200, right: ERCC-1, ×200). 
Table 1 Expression of Bax and ERCC-1 in the metastatic lymph nodes of 37 patients with stage II/III gastric cancer and positive occult neoplastic cells (ONCs) in their lymph node sinuses. The recurrence group comprised 28 patients and the non-recurrence group included 9 patients. Expression was classified as low $(-,+)$ or high $(++$, $+++)$.

\begin{tabular}{lllll}
\hline $\begin{array}{l}\text { Immunohistochemical staining [high-level; }(+++)(++), \text { low-level; }(+)(-)] \\
\text { Metastatic LNs }\end{array}$ & \multicolumn{3}{l}{ Bax } & ERCC-1 \\
\cline { 2 - 5 } \begin{tabular}{l} 
Total 37 cases \\
\cline { 2 - 5 }
\end{tabular} & high-level & low-level & high-level & low-level \\
\hline Recurrence $(\mathrm{n}=28)$ & $53.6 \%(15 / 28)$ & $46.4 \%(13 / 28)$ & $32.1 \%(9 / 28)$ & $67.9 \%(19 / 28)$ \\
Non-recurrence $(\mathrm{n}=9)$ & $44.4 \%(4 / 9)$ & $55.6 \%(5 / 9)$ & $33.3 \%(3 / 9)$ & $66.7 \%(6 / 9)$ \\
\hline
\end{tabular}

LNs; lymph nodes, Bax; bcl-2 associated X, ERCC-1; excision repair cross-complementation group 1

whether or not tumors were sensitive to CPT-11 and platinum derivatives, the expression of Topo-1, Bax, and ERCC-1 was investigated and the percentage of patients with high Topo-1 expression or with high Bax/low ERCC-1 expression was determined ${ }^{23-25)}$.

\section{Statistical analysis}

The $\chi^{2}$ test was used to examine the significance of differences between the recurrence and non-recurrence groups, and probability values of less than 0.05 were considered to indicate a significant difference. SPSS 13.0 J software (SPSS Japan, Inc., Tokyo, Japan) was used for all analyses.

\section{Results}

\section{(1) Immunohistochemistry for Bax/ERCC-1}

In the RG $(n=28)$, high Bax expression was observed in 15 patients $(53.6 \%)$ and low expression was found in 13 patients $(46.4 \%)$. In the N-RG $(n=9)$, high Bax expression was observed in 4 patients $(44.4 \%)$ and low expression was noted in the other 5 patients $(55.6 \%)(\mathrm{P}=$ not significant; N.S.) (Table I). With respect to ERCC-1, high expression was noted in 9 patients $(32.1 \%)$ from the RG $(n=28)$ and low expression was seen in 19 patients (67.9\%), while there was high ERCC-1 expression in 3 patients $(33.3 \%)$ from the N-RG $(n=9)$ and low expression in 6 patients $(66.7 \%)$ from the N-RG (N.S.) (Table I). There were no significant differences of Bax/ERCC-1 expression between the two groups (Table I).

\section{(2) Immunohistochemistry for Topo-1 and Bax/ ERCC-1}

In the RG ( $\mathrm{n}=28)$, high Topo-1 expression was observed in 3 patients $(10.7 \%)$ and low expression was seen in 25 patients $(89.3 \%)$, while the N-RG $(n=9)$ showed high Topo-1 expression in 1 patient $(11.1 \%)$ and low expression in 8 patients $(88.9 \%)$ (N.S.) (Table II). In the RG ( $\mathrm{n}=28)$, high Bax expression was combined with low ERCC-1 expression (high Bax/low ERCC-1 expression) in 11 patients $(39.3 \%)$ and other patterns of expression were seen in 17 patients $(60.7 \%)$, while the N-RG $(n=9)$ showed high Bax/low ERCC-1 expression in 3 patients $(33.3 \%)$ and other patterns in 6 patients $(66.7 \%)$ (N.S.)
(Table II). There was a significant difference between the rate of high-Bax/low-ERCC-1 expression and that of high Topo-1 expression in the RG ( $\mathrm{p}=0.0136)$ (Table II).

\section{Discussion}

We considered that the drug sensitivity profile of ONCs should be investigated because these cells exist in organs/tissues distant from the primary tumor and might cause recurrence/metastasis. However, we thought that it would be difficult to prepare serial sections of LNs for routine H.E. staining and then detect Topo-1 and Bax/ ERCC-1 expression in the same ONCs, so we studied the metastatic LNs instead. Topo-1 and Bax/ERCC-1 were easily detected in tumor cells, with the nucleus and cytoplasm being prominently stained, while the cell membrane, interstitial cells, non-cancerous tissue, and normal epithelium were weakly stained or unstained. Both CPT-11 and platinum derivatives are DNA synthesis inhibitors, so the enzymes relevant to their actions were well stained in the nucleus of the cancer cells and were overexpressed in the cytoplasm. For further investigation of the localization and distribution of Topo-1 and Bax/ERCC-1, immune electron microscopy may be necessary.

To perform effective postoperative adjuvant chemotherapy for the prevention of recurrence/metastasis after curative resection of a primary tumor, it is necessary to eliminate residual cancer cells that may have reached various other organs/tissues during the perioperative period. Active systemic consolidation chemotherapy during the early period after surgery is expected to be most effective for this purpose. Previous reports on the sensitivity of tumors to 5-FU $+\mathrm{LV}$ have revealed that there were not many patients with sensitive tumors, including those with high TS/low DPD expression, at the time when ONC clusters were circulating and had the potential to cause recurrence/metastasis. This suggests that 5-FU is not a sufficiently powerful drug ${ }^{30-33)}$. To solve this problem, CPT-11 and/or platinum derivatives such as CDDP, which are more widely used in the US and Europe than in Japan, may be useful as modulators of 5-FU with an additive or synergistic effect. Although the dosage/administration schedule also influences the outcome, sensitivity to the drugs used for combination 
Table 2 Topo-1 expression and the combination of high Bax/low ERCC-1 expression in the metastatic lymph nodes of 37 patients with stage II/III gastric cancer and positive occult neoplastic cells (ONCs) in their lymph node sinuses. The recurrence group comprised 28 patients and the non-recurrence group included 9 patients. Expression was classified as low $(-,+)$ or high $(++,+++)$.

\begin{tabular}{lllll}
\hline \multirow{2}{*}{$\begin{array}{l}\text { Immunohistochemical staining [high-level; }(+++)(++), \text { low-level; }(+)(-)] \\
\text { Metastatic LNs }\end{array}$} & \multicolumn{3}{c}{ Topo-1 } & \multicolumn{2}{c}{ Bax \& ERCC-1 } \\
\cline { 2 - 5 } Total 37 cases & high-level & low-level & high Bax/low ERCC-1 & Others \\
\hline Recurrence $(\mathrm{n}=28)$ & $10.7 \%(3 / 28)^{\mathrm{a}}$ & $89.3 \%(25 / 28)$ & $39.3 \%(11 / 28)$ & $60.7 \%(17 / 28)$ \\
Non-recurrence $(\mathrm{n}=9)$ & $11.1 \%(1 / 9)$ & $88.9 \%(8 / 9)$ & $33.3 \%(3 / 9)$ & $66.7 \%(6 / 9)$ \\
\hline
\end{tabular}

LNs; lymph nodes, Topo-1; topoisomerase-1 inhibitor, Bax; bcl-2 associated X, ERCC-1; excision repair cross-complementation group 1, a; $\mathrm{p}=0.0136$

therapy is presumed to be a key factor in achieving better results of chemotherapy. There were no differences of Bax/ERCC-1 expression between the recurrence and non-recurrence groups, so we next investigated the number of patients with high Bax/low ERCC-1 expression or high Topo-1 expression in the recurrence group. High Bax/low ERCC-1 expression was observed in $39.3 \%$ of the patients, while high Topo-1 expression was seen in 10.7\%. Although patients with high Bax/low ERCC-1 expression were more common than those with high Topo-1 expression in the recurrence group $(p=0.0136)$, there were no significant differences between the recurrence and non-recurrence groups. These immunohistochemical findings suggested that platinum derivatives such as CDDP are more effective than CPT-11 in both RG and N-RG of patients with stage II/III gastric cancer and $\mathrm{ONCs}^{15}$. For the treatment of distant metastasis/recurrence in organs such as the liver or lungs and to manage peritoneal dissemination, it may be reasonable to try CDDP + CPT-11 as a second-line combination without 5 -FU, if postoperative adjuvant 5-FU-based therapy is not sufficiently effective ${ }^{13,14)}$.

Obtaining a higher survival rate after recurrence/metastasis is considered to be the most important factor for improving the outcome of gastric cancer patients ${ }^{34,35)}$. For the more effective treatment of stage II/III LNpositive gastric cancer, it appears to be important to detect patients with a high risk of recurrence/metastasis and provide appropriate therapy at the stage of occult metastasis soon after curative resection and before overt recurrence. To achieve a better outcome, it also appears to be preferable to use 5-FU in combination with other more active drugs. However, further investigation of the optimum dosage and administration schedule such as continuous 5-FU infusion with plus low-dose CDDP and high-dose intravenous CDDP plus 5-FU is necessary.

\section{Acknowledgement}

This study was supported by grants from the Occult Neoplastic Cells Research and Study Group (\#2007-5007; Tokai University Hachioji Hospital, Hachioji, Tokyo, Japan) and the Research and Study Program of Tokai University Educational System General Research
Organization (\#2005-41; Tokai University Hospital, Isehara, Kanagawa, Japan).

\section{References}

1) Mukai M, Ito I, Mukoyama S, Tajima T, Saito Y, Nakasaki H, Sato $\mathrm{S}$, and Makuuchi H. Improvement of 10-year survival by Japanese radical lymph node dissection in patients with Dukes' B and C colorectal cancer: A 17-year retrospective study. Oncol Rep 10: 927-934, 2003.

2) Mukai M, Sato S, Komatsu N, Nishida T, Shiba K, Ito I, Nakasaki $\mathrm{H}$, and Makuuchi $\mathrm{H}$. Correlation between occult neoplastic cells in the lymph node sinuses and recurrence in patients with Dukes' C colorectal cancer. Oncol Rep 10: 1165-1169, 2003.

3) Mukai M, Sato S, Komatsu N, Nishida T, Shiba K, Ito I, Nakasaki $\mathrm{H}$, andMakuuchi $\mathrm{H}$. Correlation between occult neoplastic cells in the lymph node sinuses and recurrence in patients with curatively resected Dukes'B colorectal cancer. Oncol Rep 10: 1177-1181, 2003.

4) Mukai M, Sato S, Nishida T, Komatsu N, Shiba K, Nakasaki H, and Makuuchi H. Selection criteria for high risk and low risk groups of recurrence and metastasis in patients with primary colorectal cancer. Oncol Rep 10: 1753-1758, 2003.

5) Mukai M, Sato S, Nakasaki H, Saito Y, Nishiumi N, Iwasaki M, Tokuda Y, Ogoshi K, Inoue H, and Makuuchi H. Occult neoplastic cells in the lymph node sinuses and recurrence of primary breast, lung, esophageal and gastric cancer. Oncol Rep 11: 81-84, 2004.

6) Mukai M, Sato S, Komatsu N, Kimura T, Ninomiya H, Nakasaki $\mathrm{H}$, Ogoshi K, and Makuuchi M. Accuracy of criteria for predicting the recurrence and metastasis of stage I and II gastric cancer without lymph node metastasis. Oncol Rep 12: 59-62, 2004.

7) Mukai M, Sato S, Komatsu N, Kimura T, Ninomiya H, Nakasaki $\mathrm{H}$, Ogoshi K, and Makuuchi H. Predicting the recurrence/metastasis of stage II and III breast cancer with lymph node metastasis. Oncol Rep 12: 303-306, 2004.

8) Mukai M, Sato S, Tajima T, Kimura T, Komatsu N, Ninomiya H, Nakasaki H, Ogoshi K, and Makuuchi H. Predicting recurrence and metastasis of Dukes' A primary colorectal cancer with or without proper muscle invasion. Oncol Rep 12: 1305-1308, 2004.

9) TNM classification of malignant tumours (6th edition), John Wiley \& Sons, Inc., New York, USA, 2002.

10) Mukai M. Occult neoplastic cells and malignant micro-aggregates in lymph node sinuses: Review and hypothesis. Oncol Rep 14: 173-175, 2005.

11) Mukai M, Sato S, Ninomiya H, Wakui K, Komatsu N, Tsuchiya K, Tajima T, Nakasaki H, and Makuuchi H. Prospective study on the recurrence/metastasis of stage II/III colorectal and gastric cancer associated with occult neoplastic cells in lymph node sinuses: Three-year interim results. Oncol Rep 16: 405-410, 2006.

12) Mukai M, Oida Y, Tajima T, Kishima K, Ninomiya H, Sato $S$, Nakamura M, Nakasaki H, and Makuuchi H. Alternating hepatic arterial infusion and systemic chemotherapy for stage IV colorectal cancer with synchronous liver metastasis. Oncol Rep 16: 865-870, 2006.

13) Hsu C-H, Yeh k-H, Chen L-T, Liu JM, Jan C-M, Lin J-T, Chen Y-C, and Cheng A-L. Weekly 24-hour infusion of high-dose 5-fluoro- 
uracil and leucovorin in the treatment of advanced gastric cancers. Oncology 54: 275-280, 1997.

14) Chen J-S, Liu H-E, Wang C-H, Yang T-S, Wang H-M, Liau C-T, Chang W-C, and Lin Y-C. Weekly 24-H infusion of high-dose 5 -fluorouracil and leucovorin in patients with advanced gastric cancer. Anti-Cancer Drugs 10: 355-359, 1999.

15) Enjoji A and the Nagasaki digestive organ cancer chemotherapy study group. Combination chemotherapy of 5 -fluorouracil and low-dose Cisplatin in advanced and recurrent gastric cancer: a multicenter retrospective study in Nagasaki, Japan. Anticancer Res 22: 1135-1140, 2002.

16) Tournigand C, Achille TAE, Lledo G, Flesh M, Quinaux DM-ME, Couteau C, Buyse M, Ganem G, Landi B, Colin P, Louvet C, and De Gramont A. FOLFIRI followed by FOLFOX 6 or the reverse sequence in advanced colorectal cancer: A randomized GERCOR study. J Clin Oncol 22: 229-237, 2004.

17) Colucci G, Gebbia V, Paoletti G, Giuliani F, Caruso M, Gebbia N, Cartenì G, Agostara B, Pezzella G, Manzione L, Borsellino N, Misino A, Romito S, Durini E, Cordio S, Seri MD, Lopez M, and Maiello E. Phase III randomized trial of FOLFIRI versus FOLFOX 4 in the treatment of advanced colorectal cancer: A multicenter study of Gruppo Oncologico Dell'Italia Meridionale. J Clin Oncol 23: 4866-4875, 2005.

18) Dabholkar M, Vionnet J, Bostick-Bruton F, Yu JJ, and Reed E. Messenger RNA levels of XPAC and ERCC1 in ovarian cancer tissue correlate with response to platinum-based chemotherapy. J Clin Invest 94: 703-708, 1994.

19) Perego P, Giarosa M, Righetti SC, Supino R, Caserini C, Delia D, Pierotti MA, Miyashita T, Reed JC, and Zunino F. Association between cisplatin resistance and mutation of $\mathrm{p} 53$ gene and reduced bax expression in ovarian carcinoma cell systems. Cancer Res 56: 556-562, 1996.

20) Li Q, Gardner K, Zhang L, Tsang B, Bostick-Bruton F, and Reed E. Cisplatin induction of ERCC-1 mRNA expression in A2780/CP70 human ovarian cancer cells. J Biol Chem 273: 23419-23425, 1998.

21) Hector S, Bolanowska-Higdon W, Zdanowicz J, Hitt S, and Pendyala L. In vitro studies on the mechanisms of oxaliplatin resistance. Cancer Chemother Pharmocol 48: 398-406, 2001.

22) Arnould S, Hennebelle I, Canal P, Bugat R, and Guichard S. Cellular determinants of oxaliplatin sensitivity in colon cancer cell lines. Euro J Cancer 39: 112-119, 2003.

23) Mukai M, Sato S, Ninomiya H, Wakui K, Komatsu N, Matsui N, Nakamura M, Nakasaki H, and Makuuchi H. Sensitivity to CPT-11 and platinum derivatives for stage III/Dukes' C colorectal cancer with occult neoplastic cells in lymph node sinuses. Oncol Rep 17: 1027-1032, 2007.

24) Mukai M, Sato S, Ninomiya H, Wakui K, Komatsu N, Matsui N, Nakamura M, Nakasaki H, and Makuuchi H. Sensitivity to CPT-11 and platinum derivatives for stage II/Dukes'B colorectal cancer with occult neoplastic cells in lymph node sinuses. Oncol Rep 17: 1045-1050, 2007.

25) Mukai M, Sato S, Ninomiya H, Wakui K, Komatsu N, Matsui N, Nakamura M, Nakasaki H, and Makuuchi H. Sensitivity to CPT-11 and platinum derivatives of stage I/II node-negative breast, lung and gastric cancer with occult neoplastic cells in lymph node sinuses. Oncol Rep 18: 33-39, 2007.

26) Nakane PK, and Pierce GB. Enzyme-labeled antibodies: preparation and application for localization of antigens. J Histochem Cytochem 14: 929-931, 1966.

27) Nakane PK, and Pierce GB. Enzyme-labeled antibody for the light and electron microscopic localization of tissue antigens. J Cell Biol 33: 307-318, 1967.

28) Okabe H, Tsujimoto H, and Fukushima M. Preparation of the antibodies against recombinant human thymidylate synthase for the detection of its intratumoral levels and application to sensitivitystudy of 5-fluorouracil. Oncol Rep 4: 685-690, 1997.

29) Kamoshida S, Shiogama K, Matsuoka H, Matsuyama A, Shimomura $\mathrm{R}$, Inada $\mathrm{K}$, Maruta M, and Tsutsumi Y. Immunohistochemical demonstration of dihydropyrimidine dehydrogenase in normal and cancerous tissues. Acta Histochem Cytochem 36: 471-479, 2003.
30) Mukai M, Sato S, Ninomiya H, Wakui K, Komatsu N, Tsuchiya K, Nakasaki H, and Makuuchi H. Recurrence and 5-FU sensitivity of stage III/Dukes' $\mathrm{C}$ colorectal cancer with occult neoplastic cells in lymph node sinuses. Oncol Rep 14: 1165-1169, 2005.

31) Mukai M, Sato S, Ninomiya H, Wakui K, Komatsu N, Tsuchiya K, Nakasaki H, and Makuuchi H. Recurrence and 5-FU sensitivity of stage II/Dukes'B colorectal cancer with occult neoplastic cells in lymph node sinuses. Oncol Rep 14: 1171-1176, 2005.

32) Mukai M, Sato S, Ninomiya H, Wakui K, Komatsu N, Tsuchiya K, Nakasaki H, and Makuuchi H. Recurrence and 5-FU sensitivity of node-positive stage II/III gastric cancer with occult neoplastic cells in lymph node sinuses. Oncol Rep 14: 1505-1510, 2005.

33) Mukai M, Sato S, Ninomiya H, Wakui K, Komatsu N, Tsuchiya K, Nakasaki H, and Makuuchi H. Recurrence and 5-FU sensitivity of node-negative stage I/II breast, lung, or gastric cancer with occult neoplastic cells in lymph node sinuses. Oncol Rep 15: 815-820, 2006.

34) Feinstein AR, Sosin DM and Wells CK. The Will Rogers phenomenon: Stage migration and new techniques as a source of misleading statistics for survival in cancer. N Engl J Med 312: 1604-1608, 1985.

35) Bunt AMG, Hermans J, Smit VTHBM, van de Velde CJH, Fleuren GL, and Bruijn JA. Surgical/pathologic stage migration confounds comparisons of gastric cancer survival rates between Japan and Western countries. J Clin Oncol 13: 19-25, 1995. 